\title{
Azithromycin and Levofloxacin Use and Increased Risk of Cardiac Arrhythmia and Death
}

Gowtham A. Rao, MD, PbD, MPH $H^{1-4}$

Josbua R. Mann, MD, MPH'1,3

Azza Shoaibi, BPharm, MPH'1-4

Charles Lee Bennett, $M D, P b D$, $M P P^{2-4}$

Georges Nabhas, MPH

S. Scott Sutton, PharmD ${ }^{2,4}$

Sony Jacob, $M D^{2}$

Scott M. Strayer, MD, MPH

'Department of Family and Preventive Medicine, School of Medicine, University of South Carolina, Columbia, South Carolina

${ }^{2}$ William J.B. Dorn Veterans Affairs Medical Center, Columbia, South Carolina

${ }^{3}$ School of Public Health, Columbia, University of South Carolina, South Carolina

${ }^{4}$ South Carolina College of Pharmacy, Columbia, South Carolina

Conflicts of interest: authors report none.

\section{CORRESPONDING AUTHOR}

Gowtham A. Rao, MD, PhD, MPH

Department of Family and Preventive

Medicine

School of Medicine

University of South Carolina

3209 Colonial Dr

Columbia, SC 29203

gowtham.rao@va.gov

\begin{abstract}
PURPOSE Azithromycin use has been associated with increased risk of death among patients at high baseline risk, but not for younger and middle-aged adults. The Food and Drug Administration issued a public warning on azithromycin, including a statement that the risks were similar for levofloxacin. We conducted a retrospective cohort study among US veterans to test the hypothesis that taking azithromycin or levofloxacin would increase the risk of cardiovascular death and cardiac arrhythmia compared with persons taking amoxicillin.
\end{abstract}

METHODS We studied a cohort of US veterans (mean age, 56.8 years) who received an exclusive outpatient dispensation of either amoxicillin $(n=979,380)$, azithromycin $(n=594,792)$, or levofloxacin $(n=201,798)$ at the Department of Veterans Affairs between September 1999 and April 2012. Azithromycin was dispensed mostly for 5 days, whereas amoxicillin and levofloxacin were dispensed mostly for at least 10 days.

RESULTS During treatment days 1 to 5, patients receiving azithromycin had significantly increased risk of death (hazard ratio $[\mathrm{HR}]=1.48 ; 95 \% \mathrm{Cl}, 1.05-2.09$ ) and serious arrhythmia ( $\mathrm{HR}=1.77 ; 95 \% \mathrm{Cl}, 1.20-2.62)$ compared with patients receiving amoxicillin. On treatment days 6 to 10, risks were not statistically different. Compared with patients receiving amoxicillin, patients receiving levofloxacin for days 1 to 5 had a greater risk of death $(\mathrm{HR}=2.49,95 \% \mathrm{Cl}, 1.7-3.64)$ and serious cardiac arrhythmia ( $\mathrm{HR}=2.43,95 \% \mathrm{Cl}, 1.56-3.79)$; this risk remained significantly different for days 6 to 10 for both death $(\mathrm{HR}=1.95,95 \% \mathrm{Cl}, 1.32$ 2.88) and arrhythmia ( $\mathrm{HR}=1.75 ; 95 \% \mathrm{Cl}, 1.09-2.82)$.

CONCLUSIONS Compared with amoxicillin, azithromycin resulted in a statistically significant increase in mortality and arrhythmia risks on days 1 to 5 , but not 6 to 10. Levofloxacin, which was predominantly dispensed for a minimum of 10 days, resulted in an increased risk throughout the 10-day period.

Ann Fam Med 2014;12:121-127. doi: 10.1370/afm.1601.

\section{INTRODUCTION}

A zithromycin is a macrolide antibiotic commonly prescribed for outpatient treatment of respiratory infections, urinary tract infections, and sexually transmitted diseases. ${ }^{1}$ Macrolide antibiotics, primarily erythromycin and clarithromycin, are known to increase cardiac arrhythmogenic risks, including QT interval prolongation, torsades de pointes, and polymorphic ventricular tachycardia. ${ }^{2}$ Between 2001 and 2007, case reports described arrhythmias among persons receiving azithromycin. ${ }^{3-7}$ In 2012, Ray et $\mathrm{al}^{8}$ reported increased risks of cardiovascular death and all-cause mortality among adult, predominantly female, Medicaid patients in Tennessee who received azithromycin compared with amoxicillin, particularly among those with a high baseline risk for cardiovascular disease. ${ }^{9}$ Subsequently, the Food and Drug Administration (FDA) ${ }^{10}$ and pharmaceutical manufacturer issued public safety notifications warning of QT prolongation risks with azithromycin. Researchers from Denmark then reported that in comparison with penicillin $\mathrm{V}$, azithromycin use was not found to be associated with increased risks of death from cardiovascular 
causes in a general population of young and middleaged adults. ${ }^{11}$ We evaluated a national cohort of US veterans receiving care at the Department of Veterans Affairs (VA) to investigate whether cardiac arrhythmia and mortality risks were observed in a population of older male individuals receiving either azithromycin, amoxicillin, or levofloxacin.

\section{METHODS}

We used information from 14 million unique persons who received care at 140 unique VA Medical Centers and 600 community-based outpatient clinics between September 1, 1999, and April 30, 2012. National VA electronics health record data were searched to obtain individual-level information on demographics, administrative claims, vital signs, mortality, laboratory results, and pharmacy dispensation. The completeness, utility, accuracy, validity, and access methods of the available data, both pharmacologic and laboratory, are described on VA website, http://www.virec.research.va.gov.

Primary and secondary endpoints were all-cause mortality and serious cardiac arrhythmia, defined as any inpatient or emergency department encounter/ utilization for cardiac arrhythmia according to International Classification of Disease, Nintb Revision, Clinical Modification (ICD-9-CM) coding: long QT syndrome (426.82), ventricular tachycardia $(427.0,427.1$, 427.2), ventricular fibrillation (427.41), ventricular flutter (427.42), or cardiac arrest (427.5). Follow-up times were separated into the first 5 days and days 6 through 10 after antibiotics were dispensed, with day 1 being the first day the drug was dispensed. We compared patients who during the evaluation period received exclusively azithromycin, levofloxacin, or amoxicillin (including amoxicillin with clavulanate potassium) within 30 days after a VA outpatient visit.

Inclusion criteria included age between 30 and 74 years, no life-threatening noncardiovascular illness, no diagnosis of drug abuse, not residing in a nursing home during the previous year, no hospitalization in the preceding 30 days, not having received another antibiotic in the previous 29 days, and enrolled in VA care (having already experienced at least $1 \mathrm{VA}$ clinical, laboratory, or pharmacy encounter for 1 year preceding the index date). We excluded those who had more outpatient visit counts per year than 95\% of all included patients. Each patient could have more than 1 independent clinical treatment cycle as long as the cycles were, at least, 30 days apart. Each independent clinical cycle had its own 5- and 10-day follow-up period during which a patient could have developed either serious cardiac arrhythmia or sudden death, neither, or both. The 2 endpoints were ascertained and investigated in 2 separate analytical models. Thus patients who developed both endpoints were counted twice, but only once for each model. Only outpatient antibiotic dispensations were included.

Baseline comorbidity was identified from claims data from up to 1 year before the date of antibiotic dispensation, using comorbidity identification algorithms from the Agency for Healthcare Research and Quality (AHRQ) Clinical Classifications Software for ICD-9-CM. ${ }^{12}$ Infections that were considered to be indications for use of the antibiotics were classified into chronic obstructive pulmonary disease and bronchiectasis (490-496), pneumonia (480-486), other respiratory infection (470-478), ear nose throat infection (460-466), gastrointestinal infection (001-009), genitourinary infection (580-629), wound infection (800-999), and other serious infection (031-040) (Supplemental Table). Additional baseline covariates included selected laboratory results, dispensation of selected medications, and demographic information obtained from inside the Veterans Affairs Informatics and Computing Infrastructure (VINCI). In cases where laboratory results were unavailable for an patient (up to $15 \%$ of the laboratory results), single mean value imputation with indicator variable creation method was used. ${ }^{13}$ All selected baseline variable data are reported in the Supplemental Table.

Death was ascertained by the VA Vital Status File. ${ }^{14}$ Data management was performed using VINCI-hosted Microsoft SQL Server Management Studio (Microsoft Corp), and statistical analysis was conducted using SAS 9.3 (SAS Institute) VINCI Grid environment.

To control for confounding, inverse probability treatment weights (IPTW) ${ }^{15}$ were computed, with propensity scores derived by multinomial logistic modeling, for assignment into 1 of the 3 exposure groups using all baseline covariates included in the Supplemental Table. We considered this large and diverse number of covariates in the IPTW calculations to minimize residual confounding by unmeasured variables. Important covariates are demographics (race, age, sex), indication for antibiotics, comorbidities including cardiac morbidities, laboratory findings, and medication.

Kaplan Meier-survival curves were generated for both outcomes, with and without IPTW. We derived hazard ratios (HRs) for comparisons using a IPTW-weighted Cox proportional hazards model, with adjustment for multiple comparisons (Bonferroni adjustment), ${ }^{16}$ to achieve an overall $\alpha$ level of .05. The IPTW was calculated using an extensive set of covariates (Supplemental Table), including imputation indicator variables for laboratory results. To avoid bias from statistical instability caused by patients at the extremes of IPTW weightings, ${ }^{15}$ patients whose IPTW distributions fell outside 2 standard deviations 
of the smallest group were excluded. ${ }^{17}$ To evaluate the balance achieved in baseline covariates after weighting, we compared standardized differences before and after weighting with IPTW: all covariates had a standardized difference of less than 10, suggesting that the IPTW weighting achieved a balance that was comparable to what might have been achieved through random treatment allocation. ${ }^{18}$ We then generated a Cox proportional hazard regression model with and without weighting with IPTW, created survival curves, and estimated hazard ratios. All reported $P$ values are two-sided. Hazard ratios were computed separately for day 1 through 5 and days 6 through 10 using extended Cox regression with heavyside functions, a model that allows step functions and thus provides constant hazard ratios within different time intervals. ${ }^{19}$

The study was approved by the WJB Dorn Veterans Affairs Medical Center Institutional Review Board and other VA national oversight organizations.

\section{RESULTS}

More than 1.6 million unique antibiotics were dispensed: 979,380 amoxicillin, and 594,792 azithromycin (Supplemental Table). The entire cohort of patients had a mean age of 56.5 years, $71 \%$ were white and $18.4 \%$ were African American, $88 \%$ were male, and $78 \%$ had a history of current or prior tobacco use. The 3 exposure groups appeared similar at baseline with respect to chronic obstructive pulmonary disease (1.1\%), diabetes mellitus (4.5\%), essential hypertension $(5.0 \%)$, history of atrial fibrillation $(0.5 \%)$, and chronic renal failure $(0.2 \%)$. Laboratory values were also similar, including mean albumin, alanine transaminase, aspartate transaminase, and serum creatinine levels. Any baseline imbalance was balanced by weighting with IPTW, using more than 50 different covariates (all variables reported in the Supplemental Table).
The most frequent duration of treatment with amoxicillin was for 10 days (57.9\%) compared with 5 days $(81.0 \%)$ for azithromycin; other durations for amoxicillin were for 7 days (20.3\%) and 14 days (11.1\%). For azithromycin durations were for 4 days $(12.1 \%)$; and for levofloxacin durations were for 3 days (9.9\%), 5 days $(6.5 \%), 7$ days $(20.2 \%), 10$ days $(42.2 \%)$, and 14 days (12.9\%). For the entire cohort, the most common indications for antibiotic treatment were chronic obstructive pulmonary disease (14\%), pneumonia (1\%), other respiratory infections (11\%), ear-nose-throat infection $(29.3 \%)$, and genitourinary infection $(7.2 \%)$. For azithromycin and amoxicillin, the most common indication was ear-nose-throat infection $(42.9 \%$ and $24.0 \%$, respectively), whereas for levofloxacin it was genitourinary infection (27.8\%). The indication for use of antibiotic was part of the IPTW computation and was thus statistically balanced after weighting.

Tables 1 and 2 report the weighted hazard ratios for all-cause mortality and serious cardiac arrhythmia by antibiotic dispensed. Figures 1 through 4 illustrate the crude and the adjusted incidence rates for serious cardiac arrhythmias and all-cause death for the 10 days' follow-up period, respectively. On weighted analysis deaths per million antibiotics dispensed at the end of days 5 and 10 were, respectively for each drug, amoxicillin (154 and 324), azithromycin (228 and 422) and levofloxacin (384 and 714). At days 1 to 5, compared with amoxicillin, treatment with azithromycin had a 1.48 -fold increased risk of death $(95 \% \mathrm{CI}, 1.05$ 2.09) (Figure 2) and a 1.77-fold increased risk of development of serious arrhythmias (95\% CI, 1.56-3.79)

\section{Table 2. Weighted Hazard Ratio (Adjusted 95\% Cl) for Multiple Comparisons of Serious Arrhythmia Events Among the 3 Antibiotic Groups}

\begin{tabular}{|c|c|c|c|c|}
\hline \multirow[b]{2}{*}{ Antibiotic } & \multicolumn{2}{|c|}{ Days 1 to 5} & \multicolumn{2}{|c|}{ Days 6 to 10} \\
\hline & Amoxicillin & Azithromycin & Amoxicillin & Azithromycin \\
\hline Azithromycin & $\begin{array}{c}1.48(1.05-2.09) \\
P=.003\end{array}$ & 1 & $\begin{array}{c}1.14(0.81-1.62) \\
P=.812\end{array}$ & 1 \\
\hline Levofloxacin & $\begin{array}{c}2.49(1.70-3.64) \\
P \leq .001\end{array}$ & $\begin{array}{c}1.68(1.15-2.47) \\
P \leq .001\end{array}$ & $\begin{array}{c}1.95(1.32-2.88) \\
P \leq .001\end{array}$ & $\begin{array}{c}1.71(1.15-2.55) \\
P=.002\end{array}$ \\
\hline
\end{tabular}

\begin{tabular}{|c|c|c|c|c|c|c|}
\hline \multirow[b]{2}{*}{ Antibiotic } & \multicolumn{3}{|c|}{ Days 1 to 5} & \multicolumn{3}{|c|}{ Days 6 to 10} \\
\hline & Amoxicillin & Azithromycin & Levofloxacin & Amoxicillin & Azithromycin & Levofloxacin \\
\hline Amoxicillin & 1 & $\begin{array}{c}0.56(0.38-0.83) \\
P \leq .001)\end{array}$ & $\begin{array}{c}0.41(0.26-0.64) \\
P \leq .001\end{array}$ & 1 & $\begin{array}{c}0.73(0.49-1.10) \\
P=.179\end{array}$ & $\begin{array}{c}0.570 .35-0.92) \\
P=.039\end{array}$ \\
\hline Azithromycin & $\begin{array}{c}1.77(1.20-2.62) \\
P \leq .001\end{array}$ & 1 & $\begin{array}{c}0.73(0.47-1.13) \\
P=.315\end{array}$ & $\begin{array}{c}1.37(0.91-2.05) \\
P=.179\end{array}$ & 1 & $\begin{array}{c}0.78(0.48-1.26) \\
P=.843\end{array}$ \\
\hline Levofloxacin & $\begin{array}{c}2.43(1.56-3.79) \\
P \leq .001\end{array}$ & $\begin{array}{c}1.37(0.88-2.13) \\
P=.315\end{array}$ & 1 & $\begin{array}{c}1.75(1.09-2.82) \\
P=.039\end{array}$ & $\begin{array}{c}1.28(0.79-2.08) \\
P=.843\end{array}$ & 1 \\
\hline
\end{tabular}


Figure 1. Cumulative incidence of all-cause death among patients by antibiotic type over 10 days (crude).

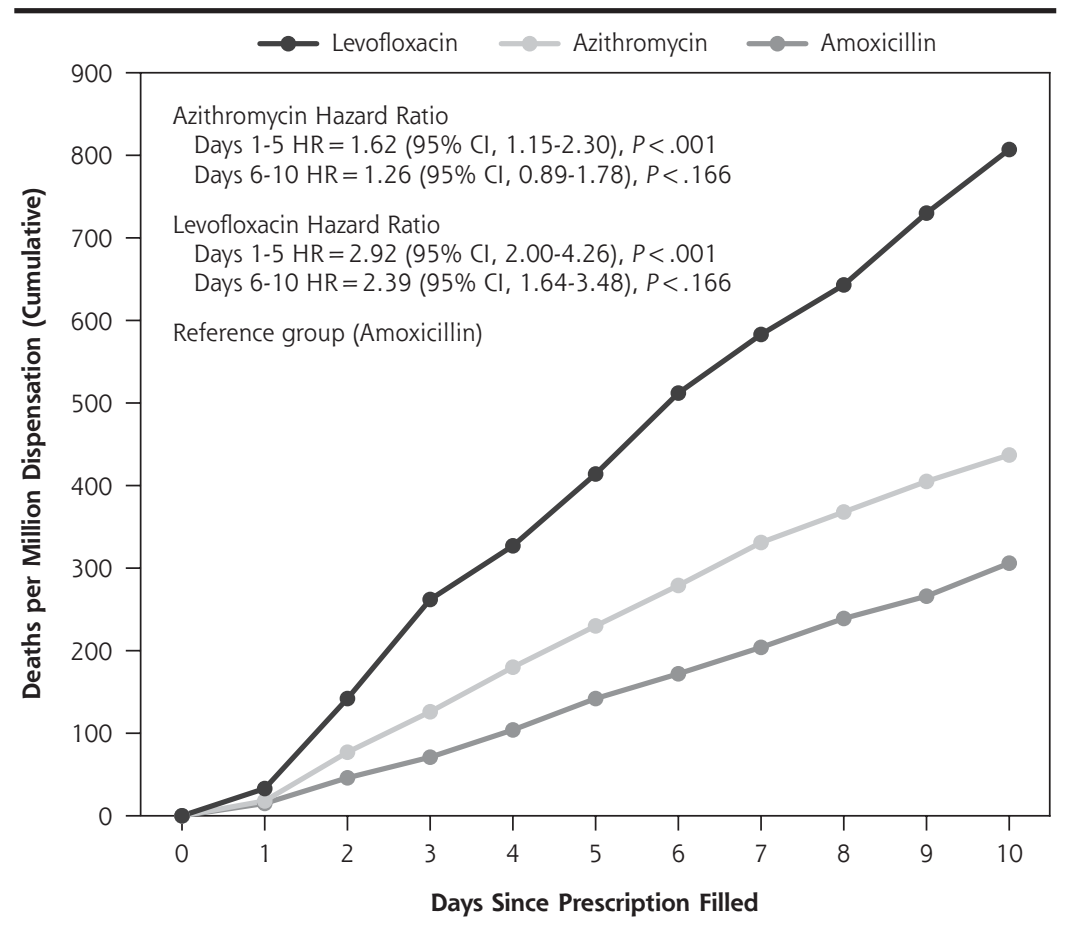

$\mathrm{HR}=$ hazard ratio

Figure 2. Cumulative incidence of all-cause death among patients by antibiotic type over 10 days (IPTW).

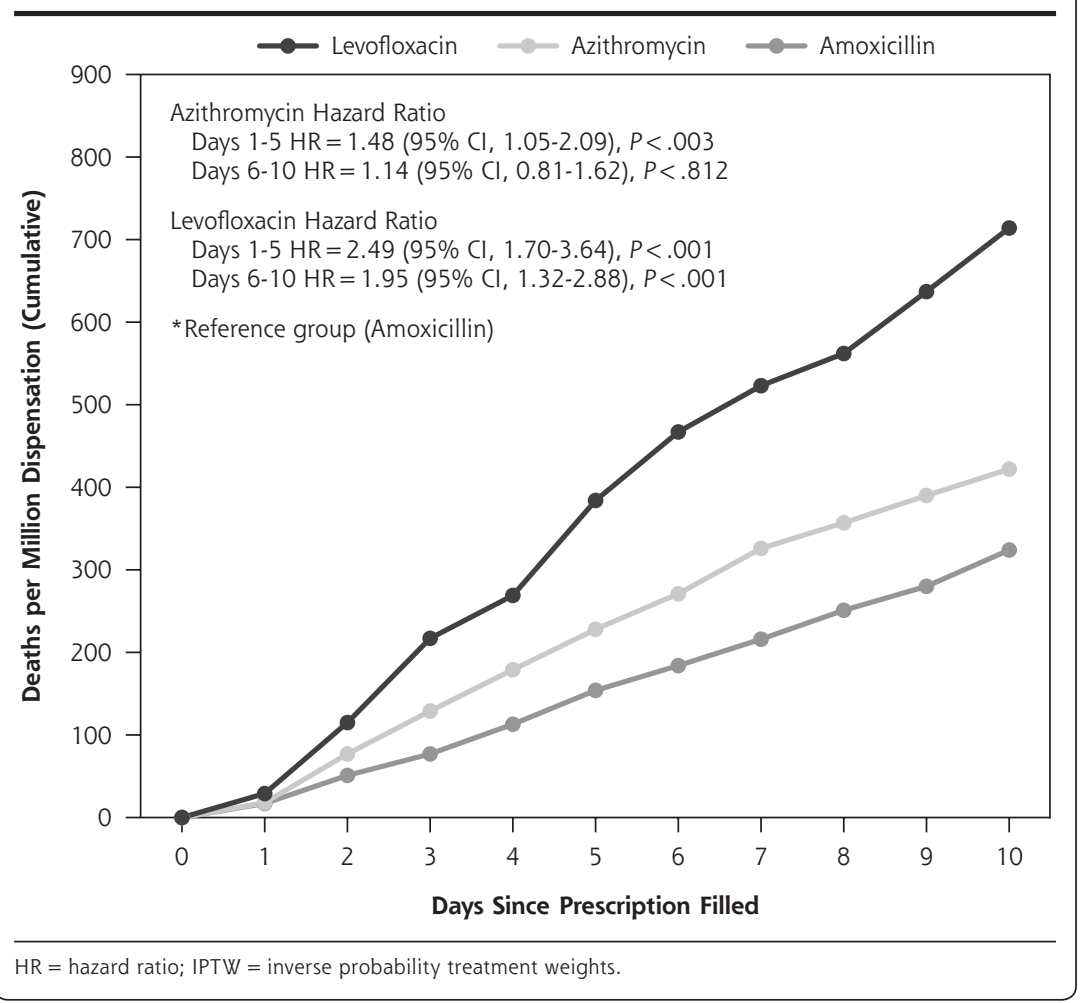

(Figure 4). At days 6 to 10, risks of death and serious arrhythmia were similar for azithromycin and amoxicillin (hazard ratio $[\mathrm{HR}]=1.14,95 \% \mathrm{CI}, 0.81-1.62$, and $\mathrm{HR}=1.37,95 \% \mathrm{CI}, 0.91-2.05$, respectively) but remained statistically significant for levofloxacin $(\mathrm{HR}=1.75,95 \% \mathrm{CI}, 1.09-2.82)$.

\section{DISCUSSION}

In this nationwide cohort study of US veterans, compared with amoxicillin, we found that a short-course of azithromycin therapy was associated with statistically significant hazard ratios of 1.47 for mortality risks and 1.77 serious arrhythmias risks within the first 5 days of treatment. The risk of these events was not significantly increased for days 6 to 10 . Treatment with levofloxacin, also when compared with amoxicillin, had statistically significant hazard ratios of 2.49 for mortality risk and 2.43 for serious arrhythmia risk; however, the increased risk with levofloxacin continued to be statistically significant during days 6 to 10 . These 2 findings, when taken in context of the traditional duration of drug treatment and the most common duration of antibiotic dispensed in our cohort, support the hypothesis of shortterm increased risk during the dispensation cycle of the drug, ie, for azithromycin 5 days, for levofloxacin at least 10 days when compared with amoxicillin.

Our study provides contextual insights into recently reported relationships of azithromycin with arrhythmia and sudden death. Ray et al reported that in comparison with short courses of amoxicillin, short courses of azithromycin were associated with 2.49 -fold (95\% CI, 1.38-4.50) increased risk of cardiovascular death and 2.02-fold 


\section{Figure 3. Cumulative incidence of serious cardiac arrhythmias among} patients by antibiotic type over 10 days (crude).

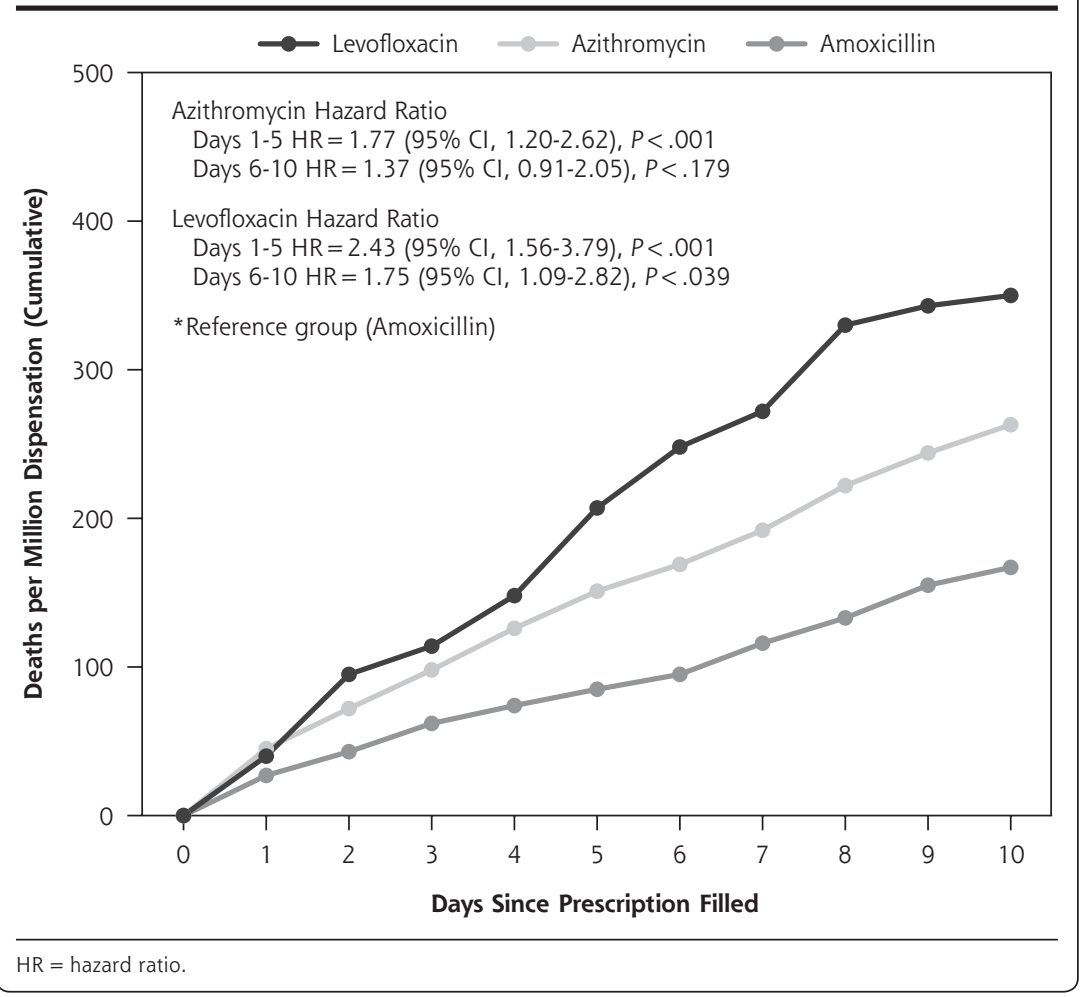

Figure 4. Cumulative incidence of serious cardiac arrhythmias among patients by antibiotic type over 10 days (IPTW).

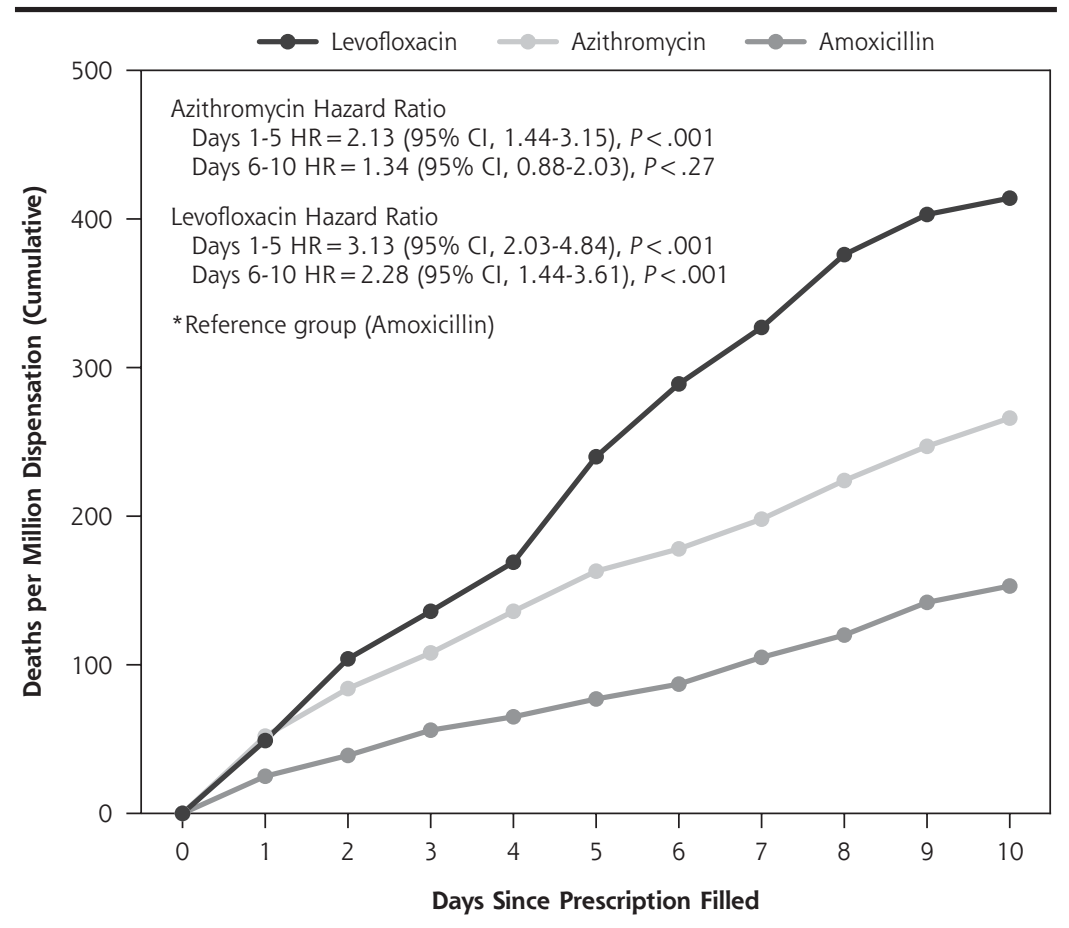

HR = hazard ratio; IPTW = inverse probability treatment weights
(95\% CI, 1.24-3.30) increased risk of death from any cause. ${ }^{8}$ The results of the Danish report using data from Denmark found that in comparison with penicillin $\mathrm{V}$, azithromycin use was not associated with an increased risk of death from cardiovascular causes. This disagreement (with our findings and the findings of Ray et al) may be due to the difference in the average age and sex composition of the studied populations. The mean age of the predominantly women cohort of the study by Ray et al was 49 years, whereas the Denmark cohort were aged a mean of 40 years (mostly young or of early middle age). Our VA cohort on other hand was that of an older male population (mean age, 56 years). In addition, the Denmark cohort is population-wide, whereas Ray et al used a specialized population of Medicaid recipients, and we used a VA population. These specialized populations may have a higher disease burden, especially cardiovascular disease, compared with the general population of Denmark. Taken together, the studies suggest that short courses of azithromycin may be associated with development of serious arrhythmias or sudden death in certain populations.

Our results provide support for recent safety announcements from the manufacturer and the Food and Drug Administration (FDA). ${ }^{10}$ In 2011, more than 40 million persons in the United States received an outpatient prescription for azithromycin. Postmarketing surveillance reports, as well published studies, found cardiovascular risks, and the FDA approved revisions to azithromycin product labels regarding risks of QT prolongation. In March 2013, the FDA announced its warning was supported by results 
of a clinical QT interval study conducted by the manufacturer of azithromycin, which found that azithromycin prolonged the QT interval. ${ }^{10}$

The strengths of this study include a very large sample size, availability of electronic health records in addition to administrative claims data, a nationwide population, and the availability of actual pharmacy dispensation data (vs prescriptions). The analytic approach of a IPTW-extended Cox proportional hazards model was similar to the design used in studies from Tennessee and Denmark. An additional benefit was adjustment for baseline laboratory values, smoking history, and body mass index, data that are not available in previous studies. These factors, along with different characteristics of the study cohort (predominantly male, older, sicker), ${ }^{20}$ allow for additional generalizability. Further, we believe that restriction to patients receiving outpatient antibiotics introduces more homogeneity to the analysis and thus validity, because patients receiving outpatient antibiotics are more likely to have similar acute conditions (vital signs, temperature, etc) compared with those not receiving antibiotics. It must be kept in mind, however, that we investigated only 3 specific antibiotics. We cannot determine from this study which alternative antibiotics might be safer. These findings must be considered in the context of an observational study, and random-allocation experimental studies to evaluate the observed outcome may not be ethical.

Residual unmeasured confounding may exist. Specifically, patients given a prescription for azithromycin or levofloxacin may be different from those who were not in ways that could bias the results. Such differences or biases may be related to the antibiotics used (confounding by indication), severity of the disease, and comorbidities. In an attempt to control for confounding by indication, our investigation included 3 different antibiotics; amoxicillin, azithromycin, and levofloxacin. Amoxicillin, the reference antibiotic, has indications similar those for azithromycin and has not been shown to have adverse cardiac effects. The indications for levofloxacin overlap those of azithromycin. The Supplemental Table, however, indicates some differences in the 3 antibiotic indications (for example, there are higher rates of chronic obstructive pulmonary disease and pneumonia as indications for azithromycin and levofloxacin and higher rates of genitourinary infections as the indication for levofloxacin). These differences are consistent with common medical practice. Different reasons for the use of the antibiotics may explain the differences in mortality observed; however, our IPTW statistical model does adjust for the indication of the antibiotic. Patients with high-risk comorbidities or/and higher disease severity may be more likely to be given prescriptions for such broadspectrum antibiotics as levofloxacin and azithromycin, which might bias the mortality results away from the null. Even so, considering the number and diversity of the covariates, balanced with IPTW, including antibiotics indication, comorbidities, variety of laboratory test results, and medications, the effect of possible residual imbalance is minimized. In addition, our exclusion criteria were formulated to exclude persons at high risk for death from causes unrelated to a short-term effect of an arrhythmia-inducing medication, which could minimize the effect of the disease severity.

Risks and benefits of antibacterial therapies should be considered when making prescription decisions. As compared with amoxicillin, there was higher risk of death associated with azithromycin therapy administered to US veterans. There are usually multiple antibiotic choices available for older patients, especially those with cardiac comorbidities; physicians may consider prescribing medications other than azithromycin and levofloxacin.

To read or post commentaries in response to this article, see it online at http://www.annfammed.org/content/12/2/121.

Key words: azithromycin; levofloxacin; amoxicillin; arrhythmia; cause of death

Submitted May 16, 2013; submitted, revised, August 30, 2013; accepted September 12, 2013.

Disclaimer: The views expressed in this article are those of the authors and do not necessarily reflect the position or policy of the Veterans Affairs or the United States Government.

Supplementary materials: Available at http://www.AnnFamMed. org/content/12/2/121/suppl/DC1/

\section{References}

1. Sanford Guide to Antimicrobial Therapy. 43rd ed. Sperryville, VA: Antimicrobial Therapy, Inc; 2013.

2. Bril F, Gonzalez CD, Di Girolamo G. Antimicrobial agents-associated with QT interval prolongation. Curr Drug Saf. 2010;5(1):85-92.

3. Huang $\mathrm{BH}$, Wu CH, Hsia CP, Yin Chen C. Azithromycin-induced torsade de pointes. Pacing Clin Electrophysiol. 2007;30(12):1579-1582.

4. Kezerashvili A, Khattak H, Barsky A, Nazari R, Fisher JD. Azithromycin as a cause of QT-interval prolongation and torsade de pointes in the absence of other known precipitating factors. J Interv Card Electrophysiol. 2007;18:243-246.

5. Matsunaga N, Oki Y, Prigollini A. A case of QT-interval prolongation precipitated by azithromycin. N Z Med J. 2003;116(1185):U666.

6. Russo V, Puzio G, Siniscalchi N. Azithromycin-induced QT prolongation in elderly patient. Acta bio-medica. Atenei Parmensis. 2006;77 (1):30-32.

7. Samarendra P, Kumari S, Evans SJ, Sacchi TJ, Navarro V. QT prolongation associated with azithromycin/amiodarone combination. Pacing Clin Electrophysiol. 2001;24(10):1572-1574. 
8. Ray WA, Murray KT, Hall K, Arbogast PG, Stein CM. Azithromycin and the risk of cardiovascular death. N Engl J Med. 2012;366(20): 1881-1890.

9. Wynn RL. Azithromycin associated with a small increased risk of cardiovascular death: a review. Gen Dent. 2013;61(2):8-9.

10. FDA Drug Safety Communication. Azithromycin (Zithromax or Zmax) and the risk of potentially fatal heart rhythms. http://www. fda.gov/drugs/drugsafety/ucm341822.htm. Accessed Mar 12, 2013

11. Svanström $H$, Pasternak $B$, Hviid A. Use of azithromycin and death from cardiovascular causes. N Engl J Med. 2013;368(18):1704-1712.

12. Clinical Classifications Software (CCS) for ICD-9-CM. HCUP CCS. Healthcare Cost and Utilization Project (HCUP). March 2013. Agency for Healthcare Research and Quality, Rockville, MD. http:// www.hcup-us.ahrq.gov/toolssoftware/ccs/ccs.jsp. Accessed May 2013.

13. Faries DE, Leon AC, Haro JM, Obenchain RL. Analysis of Observational Health Care Data Using SAS. Cary, NC: SAS Institute; 2010.

14. Sohn M-W, Arnold N, Maynard C, Hynes DM. Accuracy and completeness of mortality data in the Department of Veterans Affairs. Popul Health Metr. 2006;4:2.
15. Sugihara M. Survival analysis using inverse probability of treatment weighted methods based on the generalized propensity score. Pharm Stat. 2010;9(1):21-34.

16. Morgan JF. P Value fetishism and use of the Bonferroni adjustment. Evid Based Ment Health. 2007;10(2):34-35.

17. Kurth T, Walker AM, Glynn RJ, et al. Results of multivariable logistic regression, propensity matching, propensity adjustment, and propensity-based weighting under conditions of nonuniform effect. Am J Epidemiol. 2006;163(3):262-270.

18. Austin PC, Grootendorst P, Anderson GM. A comparison of the ability of different propensity score models to balance measured variables between treated and untreated subjects: a Monte Carlo study. Stat Med. 2007;26(4):734-753.

19. Ziegler A, Lange S, Bender R. Uberlebenszeitanalyse: Die CoxRegression. (in German). [Survival analysis: Cox regression]. Dtsch Med Wochenschr. 2007;132(Suppl 1):e42-e44.

20. Rao GA, Pandya PK. Statin therapy improves sustained virologic response among diabetic patients with chronic hepatitis C. Gastroenterology. 2011;140:144-52.e2.

\section{CHANGE-OF-ADDRESS FORM FAMAILY MEDICINE}

Please complete this form and mail to the following address or fax to Annals Circulation at 913-906-6080:

Annals of Family Medicine, Circulation Department, 11400 Tomahawk Creek Pkwy, Leawood, KS 66211-2680

Check if member of sponsoring organization:

$\begin{array}{ll}\square \text { AAFP } & \square \text { ABFM } \square \text { STFM } \square \text { ADFM } \\ \square \text { AFMRD } & \square \text { NAPCRG } \square \text { CFPC }\end{array}$

ID number from label on your journal cover

OLD Information (Please print.)

\begin{tabular}{lc}
\hline Name \\
\hline Company (if applicable) \\
\hline Address (Street plus Apt or Ste) \\
\hline City \\
\hline Country \\
\hline Telephone \\
\hline E-Mail & Faxtal Code (9-digit ZIP for US) \\
\hline
\end{tabular}

NEW Information (Please print.)

\begin{tabular}{ll}
\hline Name \\
\hline Company (if applicable) \\
\hline Address (Street plus Apt or Ste) \\
\hline City & Postal Code (9-digit ZIP for US) \\
\hline Country & Fax \\
\hline Telephone & \\
\hline E-Mail &
\end{tabular}

